\title{
50. Intensive Gare and Surgical Intervention of Cerebral Apoplexy at Acute Phase
}

\author{
Masayoshi Kowada, M. D., Shigeru Matsuoka, M. D., \\ Zentaro Ito, M. D., Yoji Gito, M.D. \\ and Akihiko Hirayama, M. D. \\ Division of Surgical Neurology, Research Institute of \\ Brain and Blood Vessels, Akita
}

An intensive care unit with an ambulance has been designed. 205 patients of cerebral apoplexy have been transfered past two years and 104 cases out of them had intensive cares at the early phase.

The level of consciousness on admission was semicoma in $37.0 \%$, coma in $22 \%$ and deep coma in $16.8 \%$. Mortality within one month after onset was $41.6 \%$ in cerebral hemorrhage, $51.5 \%$ in subaracnoid hemorrhage and $38.9 \%$ in cerebral infarction. Causes of death have been due to severly damaged brains themselves, and death by complications has been rare.

An early surgical intervention has been carried out in patients of hypertensive intracerebral hemorrhage. Twenty-one cases have been operated within 24 hours after onset and the operative mortality has been 5 per cent within one month after the removal of hematoma.

Leakages of contrast medium have been demonstrated in 5 cases, in which serial carotid angiographies have been performed within 5 hours after onset. Removals of hematoma have been carried out in 4 out of 5 cases, saving all of them.

Surgical indications have been also discussed in detail in recurred cases of cerebral apoplexy and in the hypertensive intracerebral hemorrhage with silent aneurysms.

\section{The Operative Criteria for Hemiplegia of Hypertensive Intracerebral Hemorrhage}

\author{
Masahiro Mizukami, Takashi Tomita* \\ Goro Araki and Hiroshi Mrhara \\ Division of Neurosurgery. Department of Surgery, \\ Keio University School of Medicine \\ Institute of Brain and Blood Vessel \\ Mihara Memorial Hospital
}

We have operated 53 cases of putaminal hemorrhage which was the predilection site in hypertensive intracerebral hemorrhage and correlated the prognosis for 